BULL. AUSTRAL. MATH. SOC.

VOL. $28(1983), 101-110$.

\title{
ON GROUPS WITH SMALL ENGEL DEPTH
}

\section{ROLF BRANDL}

\begin{abstract}
Every finite group $G$ satisfies a law $\left[x, x^{y}\right]=\left[x, s^{y}\right]$ for some positive integers $r<s$. The minimal value of $r$ is called the depth of $G$. It is well known that groups of depth 1 are abelian. In this paper we prove the following. Let $G$ be a finite group of depth 2 . Then $G / F(G)$ is supersoluble, metabelian and has abelian Sylow p-subgroups for all odd primes $p$. Moreover, $z_{p}(G) \leq 1$ for $p$ odd and $z_{2}\left(G^{2}\right) \leq 1$.
\end{abstract}

\section{Introduction}

If $G$ is a finite group, then there exist positive integers $r<s$ such that for all $x, y \in G$ the following holds: $\left[x, r^{y}\right]=\left[x, s^{y}\right]$. If $r$ is chosen minimal with respect to this property, we call $r$ the (Engel-) depth of $G$. Let $V_{r}$ be the class of all finite groups of Engel depth less than or equal to $r$. Obviously, a finite nilpotent group belongs to $v_{r}$ if and only if it satisfies the rth Engel condition.

In [7, Theorem 3.2] it has been proved that groups in $V_{1}$ are abelian. By contrast, the groups $\operatorname{PSL}(2,5)$ and $\operatorname{PSL}(2,8)$ are of depth 3 (D. Nikolova, Personal Communication).

Here we consider groups of depth 2 . It turns out that these groups are soluble. More precisely, we shall prove

THEOREM. Let $G$ be a finite group of depth 2 . Then

Received 2 May 1983. 
(a) $G / F(G)$ is supersoluble, metabelian and for all odd primes $p$ the sylow p-subgroups of $G / F(G)$ are abelian,

(b) if $p$ is an odd prime, $z_{p}(G) \leq 1$; also $z_{2}\left(G^{2}\right) \leq 1$.

Unless otherwise stated, all groups considered in this paper are finite.

\section{The structure of groups in $v_{2}$}

This section is devoted to a proof of the main theorem mentioned in the introduction. We first note a simple observation that turns out to be very useful in the proofs.

LEMMA 1 . Let $H \in V_{2}$ and let $A$ be a nilpotent normal subgroup of $H$. Then for each $a \in A$ the normal closure $\left(a^{H}\right\rangle$ is abelian.

Proof. Let $b \in H$. By assumption, we have $\left[b, 2^{a}\right]=\left[b, 2+k^{a}\right]$ for some $k$. So $\left[b, 2^{a}\right]=[b, 2+k t a] \in \gamma_{k t+2}(A)$ for all positive integers $t$. As $A$ is nilpotent, we get $\left[b, 2^{a}\right]=1$ and so $\left[a, a^{b}\right]=1$. This implies that $\left(a^{H}\right)$ is abelian.

We now prove that all groups in $V_{2}$ are soluble (this fact has been found independently by D. Nikolova). In order to do this, we examine the minimal simple groups (see [11]).

LEMMA 2. The Suzuki groups $\mathrm{Sz}(q)$ and $\operatorname{SL}(3,3)$ do not belong to $v_{2}$

Proof. Let $G=\mathrm{Sz}(q)$, let $A$ be a Sylow 2-subgroup of $G$ and let $H=N_{G}(A)$. Any element in $H$ of order $q-1$ acts transitively on $(A / \Phi(A))^{\#}$ and so for any $a \in A \backslash \Phi(A)$ we have $A=\left(a^{H}\right)$. But $A$ is nonabelian and so $H \notin V_{2}$ by Lemma 1 . This proves $G \notin V_{2}$.

The group $\operatorname{SL}(3,3)$ contains a subgroup $H$ isomorphic with $\mathrm{SL}(2,3)$. The same argument yields $\operatorname{SL}(3,3) \notin V_{2}$.

We now deal with the remaining minimal simple groups $G=\operatorname{PSL}(2, q)$. 
The search for suitable elements proving $G \leqslant V_{2}$ has been eased considerably by computer calculations performed on a TR440 at the Rechenzentrum der Universität Würzburg.

LEMMA 3. Let $q \geq 4$ be a prime power. Then $\operatorname{PSL}(2, q) \neq V_{2}$.

Proof. Because of the isomorphism $\operatorname{PSL}(2,5) \cong \operatorname{PSL}(2,4)$ we may assume $q \neq 5$. Let $e \in \mathrm{GF}(q)$ with $e^{2} \neq \pm 1$. Let

$$
x=\left(\begin{array}{cc}
-e^{2}\left(e^{2}+1\right)\left(e^{2}-1\right)^{-1} & \left(e^{2}-1\right)^{-3} \\
-e^{2}\left(e^{2}-1\right)^{2} & e^{-2}\left(e^{2}+1\right)^{-1}
\end{array}\right)
$$

and

$$
y=\left(\begin{array}{cc}
e^{-1} & 0 \\
0 & e
\end{array}\right)
$$

A straightforward computation yields

$$
\left[x, 2^{y}\right]=\left(\begin{array}{cc}
e^{-2} & e^{-2}\left(e^{2}-1\right)^{-1} \\
0 & e^{2}
\end{array}\right) \text {. }
$$

So for any $k \geq 3$, we have $\left[x, k^{y}\right]=\left(\begin{array}{ll}1 & * \\ 0 & 1\end{array}\right)$.

As $e^{2} \neq \pm 1$ we have shown $\left[x, 2_{2}^{y}\right] \neq \pm\left[x, k^{y}\right]$ for all $k \geq 3$. Hence $\operatorname{PSL}(2, q) \notin V_{2}$.

We now prove the first part of our main theorem.

THEOREM A. Let $G \in V_{2}$. Then $G / F(G)$ is supersoluble.

Proof. Let $G$ be a minimal counterexample. Lemma 2, Lemma 3 and [11] imply that $G$ is soluble. By [2, 2.9] we know that $G$ is a split extension of its unique minimal normal subgroup $N$ by a complement $Q$ and all proper subgroups of $Q$ are supersoluble. From [5] we infer that $Q$ has a unique normal sylow subgroup $A$ possessing a complement $B$ in $Q$. Moreover, $A / \Phi(A)$ is an irreducible $B$-module and $A$ is noncyclic. Also $\Phi(A) \leq Z(A)$.

We first show that $A$ is elementary abelian. Let $a \in A \backslash \Phi(A)$. By 
Lemma 1 we know that $\left\langle a^{B}\right\rangle$ is abelian. As $B$ acts irreducibly on $A / \Phi(A)$, we have $A=\left\langle a^{B}\right\rangle \cdot \Phi(A)=\left\langle a^{B}\right\rangle$ and so $A$ is abelian. The proof of part (f) of [5, Satz 1$]$ now yields that $A$ is elementary.

Let $l \neq a \in A$ and let $n \in N$ and $b \in B$ be arbitrary. Then $[b, n a]=[b, a][b, n]^{a}$ and so

$$
\begin{aligned}
{\left[b, 2^{n a}\right] } & =\left[[b, a][b, n]^{a}, n a\right] \\
& =[b, a, n a]^{[b, n]^{a}}\left[[b, n]^{a}, n a\right] \\
& =\left([b, a, a][b, a, n]^{a}\right)^{[b, n]^{a}}\left[[b, n]^{a}, a\right] \\
& =[b, a, n]^{a}[b, n, a]^{a}
\end{aligned}
$$

as $[b, a, a]=1$.

From $\left[b, 2^{n a}\right] \in N$ we obtain by a straightforward computation

$$
\left[b, 2+k^{n a}\right]=\left[b, a, n, k^{a}\right]^{a}\left[b, n, a, k^{a}\right]^{a} .
$$

As $G \in V_{2}$, there exists some $k$ with

$$
[b, a, n] \cdot[b, n, a]=\left[b, a, n, k^{a}\right]\left[b, n, a, k^{a}\right] .
$$

In particular, we get

$$
[b, a, n][b, n, a] \in[N, a]
$$

and so

$$
[b, a, n] \in[N, a] \text {. }
$$

Hence $[n,[b, a]]=\left[n, a^{-b} a\right] \in[N, a]$ and finally $\left[n, a^{-b}\right] \in[N, a]$. As $n \in N$ has been chosen arbitrarily, we get $\left[N, a^{-b}\right] \leq[N, a]$. The latter holds for any $b \in B$ and so $\left[N, a^{-b} 1 \ldots a^{-b} t\right] \leq[N, a]$ for all choices $b_{i} \in B$. As $B$ acts irreducibly on $A$, we have $A=\left\langle a^{B}\right\rangle$ and so we arrive at $N=[N, A] \leq[N, a]$. This implies $C_{N}(a)=1$. Hence every nonidentity element of $A$ acts fixed point freely on $N$ and so $A$ is cyclic. This, however, contradicts the structure of $A$. 
Using Theorem $A$, we can now prove

THEOREM B. Let $G \in V_{2}$. Then for all odd primes $p$, the quotient $G / F(G)$ has abelian Sylow p-subgroups.

Proof. Let $p$ be an odd prime and let $G$ be a counterexample of least possible order. From [2, 2.9] we infer that $G$ is a split extension of a uniquely determined minimal normal subgroup $N=F(G)$ by a complement $Q$. Moreover, all proper subgroups of $Q$ have abelian Sylow $p$-subgroups. This implies that $Q$ is a nonabelian $p$-group all of whose proper subgroups are abelian. So $Q$ is nilpotent of class two by a result of Rede $i$ [8, p. 309]. Also, $N$ is a $p^{\prime}$-group.

We claim that every nonidentity element of $Q$ acts fixed point freely on $N$. Indeed, let $1 \neq b \in Q$ with $C_{N}(b) \neq 1$ be given. As $Q$ acts faithfully and irreducibly on $N$, we have $b \notin Z(Q)$. So there exists $a \in Q$ with $z=[a, b] \neq 1$. Moreover, $z \in Z(Q)$.

Let $n \in C_{N}(b)$. We now compute $\left[a, k^{n b}\right]$. First

$$
[a, n b]=2 n_{1} \text { for some } n_{1} \in N \text {. }
$$

As $Q$ is nilpotent of class two, we have $\left[a, k^{n b}\right] \in N$ for all $k \geq 2$. As $G \in V_{2}$, there exists some positive integer $d$ such that $\left[a, 2^{n b}\right]=\left[a, 2+d^{n b}\right]$. Let $n_{2}=\left[a, 1+d^{n b}\right]$. Then $\left[z n_{1}, n b\right]=\left[n_{2}, n b\right]$. Hence $z n_{1} n_{2}^{-1} \in C_{G}(n b)$.

As $n b=b n$ and the orders of $n$ and $b$ are coprime, we have $n \in\langle n b\rangle$. So $z n_{1} n_{2}^{-1}$ centralizes $n$. From this we finally get $n \in C_{N}(z)$. This implies $C_{N}(b) \leq C_{N}(z)=1$ which contradicts the choice of $b$.

From [6, Theorem 10.3.1, p. 339] we conclude that $Q$ is cyclic. This contradicts the structure of $Q$.

COROLLARY. Let $G \in V_{2}$. Then $G / F(G)$ is metabelian.

Proof. Theorem A implies that $Q=G / F(G)$ is supersoluble, and so $Q^{\prime}$ is nilpotent. By Theorem $B$, all Sylow subgroups of odd order of $Q^{\prime}$ 
are abelian. Let $S$ be a Sylow 2-subgroup of $Q$. As $G \in V_{2}, S$ satisfies the second Engel condition and so is nilpotent of class two. Hence $S^{\prime}$ is abelian. As $Q$ is 2-nilpotent, $S^{\prime}$ is a Sylow 2-subgroup of $Q^{\prime}$. So $Q^{\prime}$ is abelian and the result follows.

From this we can deduce a property of infinite soluble groups of depth two.

COROLLARY. Let $G$ be poly-(abelian or finite). Assume that for any $x, y \in G$ there exists some positive integer $s=s_{1}(x, y)>2$ such that $\left[x, 2^{y}\right]=\left[x, s^{y}\right]$. Then $G$ is (2-Engel)-by-metabelian.

Proof. Let $U$ be a finitely generated subgroup of $G$. From [4, Theorem B] we infer that $U$ is finite-by-nilpotent, and so $U$ is residually finite. Every finite quotient of $U$ belongs to the variety $\underline{\underline{V}}$ of all (2-Engel)-by-metabelian groups. This implies $U \in \underline{\underline{V}}$ and so $G \in \underline{\underline{\mathrm{V}}}$.

The remainder of our main theorem now follows from

THEOREM $C$. Let $G \in V_{2}$. Then

(a) $l_{p}(G) \leq 1$ for all odd primes $p$,

(b) $z_{2}\left(G^{2}\right) \leq 1$.

Proof. (a) Let $G$ be a counterexample of least possible order. By [8, p. 693], $G$ is a split extension of its unique minimal normal subgroup $N=F(G)$, which is a $p$-group, by a complement $Q$. By the Hall-Higman reduction (see $[1, \mathrm{p} .258]$ ), $Q$ is a split extension of a normal Sylow $q$-subgroup $A$ of $Q$ by a p-group $B$ acting irreducibly on $A / \Phi(A)$. From Theorem $A$ we infer that $Q$ is supersoluble and hence $A$ is cyclic. As all nilpotent subgroups of $G$ satisfy the second Engel condition, every p-element of $Q$ acts as a linear map on $N$ with minimal polynomial dividing $(-1+X)^{2}$. The result now follows from [6, Theorem 11.1 .1 , p. 359] as $G$ has abelian Sylow $p$-subgroups for all primes $r \neq p$.

(b) Let $F$ be the class of all extensions of groups having 2-length one by elementary abelian 2-groups. As the product of a subgroup closed saturated formation containing all nilpotent groups with any formation is 
saturated, we see that $F$ is saturated.

Let $G$ be a minimal counterexample. Again $G$ is a split extension of a minimal normal subgroup $N=F(G)$ by a complement $Q$ acting faithfully on $N$. Clearly $N$ is an elementary abelian 2-group. From Theorem A we infer that $Q$ is supersoluble so that, in particular, $Q$ is 2-nilpotent. Let $x \in Q$ be a 2-element. Then $(N, x)$ is a second Engel group and so a straightforward computation shows that $x$ is an involution. This proves $Z_{2}\left(G^{2}\right)=1$ contradicting our choice of $G$.

\section{Some groups of small depth}

In the sequel a collection of examples may be found which illustrate that some stronger versions of the above theorems cease to be true. For example, the class $v_{2}$ does not contain all metabelian groups as there are metabelian p-groups of arbitrary Engel length. However

PROPOSITION 1 ([9]). Let $G$ be an extension of an abelian normal subgroup $N$ by an abelian group $Q$. If the orders of $N$ and $Q$ are coprime, then $G \in V_{2}$.

Proof. Let $x, y \in G$. Then $N=C_{N}(y) \times[N, y]=N_{1} \times N_{2}$. We have $[x, y]=n_{1} n_{2}$ for some $n_{i} \in N_{i}$. So $\left[x, 2_{2}\right]=\left[n_{2}, y\right] \in N_{2}$. As $y$ acts fixed point freely on $N_{2}$, we infer from [3, Lemma 4] that there exists some positive integer $d=d(x, y)$ with $\left.n_{2}=\left[n_{2}, \not{d}\right]\right]$. Hence $\left[x, 2^{y}\right]=\left[x, 2+d^{\not y}\right]$. Let $D$ be the least common multiple of all $d(x, y)$. Then $\left[x, 2^{y}\right]=\left[x, 2+D^{y}\right]$ for all $x, y \in G$.

An obvious generalization of Proposition 1 to groups of higher derived length does not seem to be at hand as is shown by the following example which has been computed on a TR 440 at the Rechenzentrum der Universität wïrzburg.

EXAMPLE. Let $G$ be generated by elements $n_{1}, \ldots, n_{5}$, $a_{1}, \ldots, a_{5}, b$ subject to the following defining relations: 


$$
\begin{aligned}
& n_{i}^{3}=a_{i}^{2}=b^{5}=\left[n_{i}, n_{j}\right]=\left[a_{i}, a_{j}\right]=\left[n_{i}, a_{i}\right]=1 \text { for all } \\
& i, j \text {; } \\
& n_{i}^{a}=n_{i}^{-1} \text { for all } i \neq j \text {; } \\
& a_{i}^{b}=a_{i+1}, n_{i}^{b}=n_{i+1} \text { for } i=1, \ldots, 4 \text {; } \\
& a_{5}^{b}=a_{1} \\
& n_{5}^{b}=n_{1}
\end{aligned}
$$

Let $x=b$ and $y=n_{1} a_{1} b$. Then $\left[x, 5^{y}\right]=\left[x,{ }_{50}^{y}\right]$ but $\left[x, 4^{y}\right] \neq\left[x, k^{y}\right]$ for all $k>4$. So the depth of $G$ is at least 5 , but $G$ has derived length 3 .

Another series of groups of depth 2 may be found among Frobenius groups.

PROPOSITION 2. Let $G$ be a Frobenius group with kermel $N$ and complement $Q$. If $N$ is abelian and $Q$ is metacyclic then $G \in V_{2}$.

Proof. This follows from [3, Lemma 4].

A similar sort of argument proves that any extension of an elementary abelian 2-group by the dihedral group of order $2 p$, where $p$ is any odd prime, has depth 2 . So groups in $V_{2}$ need not be metanilpotent.

We end with some speculations concerning the general situation. In view of the first corollary to Theorem $B$ one might ask whether there is a bound $f(r)$ depending on $r$ such that for any soluble group in $v_{r}$ the quotient $G / F(G)$ has derived length less than or equal to $f(r)$. Or, in view of Theorem A, are the ranks of the chief factors of $G / F(G)$ bounded by some function of $r$ ? The answer to both questions, however, is negative in general.

EXAMPLE. Let $n$ be any positive integer. By [10] there exist finite groups of exponent 4 and derived length $n$. Let $Q$ be such a group of least possible order. Then $Z(Q)$ is cyclic and so there exists a faithful and irreducible $\mathrm{GF}(p)$ Q-module $N$ ( $p$ denotes any odd prime). Let $G$ 
be the split extension of $N$ by $Q$. Now [12] implies that $Q$ satisfies the 4th Engel condition and so an argument similar to that one used in the proof of Proposition 1 shows $G \in V_{5}$.

By an analogous construction using a split extension of some faithful and irreducible $\mathrm{GF}(q) G$-module $M$ by $G$ it is possible to disprove the second statement.

Presumably it is essential in this example that the groups under consideration are not generated by two elements. A positive answer to any of these questions for two-generator groups would establish the following

CONJECTURE. There exists a function $F$ such that every soluble group in $v_{r}$ has Fitting length at most $F(r)$.

\section{References}

[1] Rolf Brandl, "A characterization of finite p-soluble groups of p-length one by commutator identities", J. Austral. Math. Soc. Ser. A 30 (1980-1981), 257-263.

[2] Rolf Brandl, "Zur Theorie der untergruppenabgeschlossenen Formationen: Endlich Varietäten", J. Algebra 73 (1981), 1-22.

[3] Rolf Brandl, "On finite abelian-by-nilpotent groups", J. Algebra (to appear).

[4] Rolf Brandl, "Infinite soluble groups with Engel cycles; a finiteness condition", Math. 2. 182 (1983), 259-264.

[5] KIaus Doerk, "Minimal nicht überauflösbare, endliche Gruppen", Math. z. 91 (1966), 198-205.

[6] Daniel Gorenstein, Finite groups (Harper and Row, New York, Evanston, London, 1968).

[1] N.D. Gupta, "Some group laws equivalent to the commutative law", Arch. Math. (Basez) 17 (1966), 97-102.

[8] B. Huppert, Endliche Gropen I (Die Grundlehren der Mathematischen Wissenschaften, 134. Springer-Verlag, Berlin, Heidelberg, New York, 1967). 
[9] Д. Николова [D. Nikolova], "Таждества в метабелевьх многообразий $A_{k} A_{1}$ [Identities in the metabelian variety $A_{k} A_{1}$ ], Serdica (to appear).

[10] 10.П. Размыслов [ Ju.P. Razmyslov], "О гроблема Халл-Хигмен" [The Hall-Higman problem], Izv. Akad. Nauk SSSR Ser. Mat. 42 $(1978), 833-847$.

[11] John G. Thompson, "Nonsolvable finite groups all of whose local subgroups are solvable", Bul2. Amer. Math. Soc. 74 (1968), 383-437.

[12] C.R.B. Wright, "On the nilpotency class of a group of exponent four", Pacific J. Math. 11 (1961), 387-394.

\footnotetext{
Mathematisches Institut,

Am Hubland 12,

D-8700 würzburg,

Germany.
} 